\title{
Mature oligodendrocytes actively increase in vivo cytoskeletal plasticity following CNS damage
}

\author{
Giuseppe Locatelli ${ }^{1,2}$, Arianna Baggiolini ${ }^{1 \dagger}$, Bettina Schreiner ${ }^{1 \dagger}$, Pushpalatha Palle ${ }^{4,5}$, Ari Waisman $^{3}$, \\ Burkhard Becher ${ }^{1}$ and Thorsten Buch ${ }^{1,4,5^{*}}$
}

\begin{abstract}
Background: Oligodendrocytes are myelinating cells of the central nervous system which support functionally, structurally, and metabolically neurons. Mature oligodendrocytes are generally believed to be mere targets of destruction in the context of neuroinflammation and tissue damage, but their real degree of in vivo plasticity has become a matter of debate. We thus investigated the in vivo dynamic, actin-related response of these cells under different kinds of demyelinating stress.
\end{abstract}

Methods: We used a novel mouse model (oLuCR) expressing luciferase in myelin oligodendrocyte glycoproteinpositive oligodendrocytes under the control of a $\beta$-actin promoter. Activity of this promoter served as surrogate for dynamics of the cytoskeleton gene transcription through recording of in vivo bioluminescence following diphtheria toxin-induced oligodendrocyte death and autoimmune demyelination. Cytoskeletal gene expression was quantified from mature oligodendrocytes directly isolated from transgenic animals through cell sorting.

Results: Experimental demyelinating setups augmented oligodendrocyte-specific in vivo bioluminescence. These changes in luciferase signal were confirmed by further ex vivo analysis of the central nervous system tissue from oLucR mice. Increase in bioluminescence upon autoimmune inflammation was parallel to an oligodendrocytespecific increased transcription of $\beta$-tubulin.

Conclusions: Mature oligodendrocytes acutely increase their cytoskeletal plasticity in vivo during demyelination. They are therefore not passive players under demyelinating conditions but can rather react dynamically to external insults.

Keywords: Demyelination, Experimental autoimmune encephalomyelitis, In vivo imaging, CNS plasticity, Cytoskeletal dynamics

\section{Background}

Oligodendrocytes (ODCs) are cells of the central nervous system (CNS) whose processes form myelin, a multi-layered membrane structure participating in saltatory signal conduction [1] and metabolic support of neuronal axons [2,3]. Myelin is produced in the last developmental stage of ODCs through a rapid, tightly regulated process [4] in which

\footnotetext{
* Correspondence: thorsten.buch@uzh.ch

${ }^{\dagger}$ Equal contributors

${ }^{1}$ Institute of Experimental Immunology, University of Zurich,

Winterthurerstrasse 190, Zürich 8057, Switzerland

${ }^{4}$ Institute for Medical Microbiology, Immunology and Hygiene, Technische

Universität München, Trogerstrasse 30, 80675 Munich, Germany

${ }^{5}$ Institute of Laboratory Animal Science, VetSuisse, University of Zurich,

Winterthurerstrasse 190, Zurich 8057, Switzerland

Full list of author information is available at the end of the article
}

overlaying contiguous membranes become strongly interconnected by extruding cytoplasm to form compact myelin [5]. These membrane domains remain directly connected to the cell body with a complex underlying cytoarchitecture comprising microtubules distributed in larger processes and actin filaments enriched in thinner myelin domains and in paranodes [6,7]. $\beta$-actin and $\beta$-tubulin are thus main players in dynamics of axon targeting and myelin stability $[7,8]$.

Acute or chronic damage to ODCs inevitably leads to neuronal loss as observed in several animal models [9-11] and human diseases such as multiple sclerosis (MS) and inherited leukodystrophies of the CNS [10]. However, demyelination and ODC death also lead to the activation of oligodendrocyte progenitor cells (OPCs) [11-15]. These cells can develop into mature ODCs and remyelinate naked 
axons, thus restoring saltatory conduction [16]. In this context, the role of surviving mature ODCs within and surrounding damaged CNS areas is still unclear. While it is current dogma that mature ODCs lack the ability to remyelinate axons [13,17], some studies indicate that these cells can at least maintain different degrees of structural plasticity. Earlier observations in different experimental paradigms and within MS lesions indicate sparse mature ODC proliferation within remyelinating areas [18-20], and in vitro ODCs can survive complement attack by actively shedding myelin vesicles [21], regenerate myelin processes after damage [22,23], and display migratory capability after maturation [24]. Also, the fact that ODCs close to or within neuroinflammatory lesions that have been deprived of their myelin processes can survive this insult $[25,26]$ suggests the existence of active mechanisms of cellular plasticity.

Insights into dynamic properties of ODCs could come from the study of the cell cytoarchitecture which regulates and drives membrane movements [27]. In order to investigate the plasticity of mature ODCs under demyelinating conditions in vivo, we have thus generated a mouse model (oLucR) in which luciferase expression is controlled by a $\beta$-actin promoter and restricted specifically to mature ODCs [28]. CNS-specific bioluminescence in oLucR mice was quantitatively measured in vivo after injection of luciferin. We followed bioluminescence changes in two experimental models of ODC damage, namely diphtheria toxin (DTx)-mediated ODC killing (oDTR model $[11,29,30]$ ), and in the neuroinflammatory paradigm experimental autoimmune encephalomyelitis (EAE) [1]. oLucR mice revealed defined and reproducible increases in the in vivo bioluminescence during induced demyelination in both experimental paradigms, independently from de novo ODC generation from progenitors. The measured in vitro and ex vivo bioluminescence correlated with the in vivo longitudinal data, indicating that our observations revealed an intrinsic feature of the damaged ODC population. Transcriptional analysis of structural genes in the damaged CNS and specifically within ODCs showed increased expression of cytoskeleton genes after demyelinating insult. Our results thus elaborate in a novel in vivo model previous suggestions that ODCs undergoing/sensing cellular stress can transiently enhance their own plasticity [21-24]; furthermore, we provide important insights on the timing and extent of such activation in in vivo experimental demyelination models.

\section{Methods}

\section{Animals}

Mice were kept under SPF conditions according to Swiss and German animal laws and institutional guidelines. Animal experiments were conducted under the license numbers 13/2006 and 55.2-1-54-2532-1-12 after approval by the respective Swiss and German government agencies, the Veterinäramt of the Canton of Zurich and the Regierung von Oberbayern. The presence of respective transgenes was confirmed by PCR analysis on DNA from tail biopsies by the use of the following primer pairs: MOGi-cre [29] (WT 350 bp) GAC AAT TCA GAG TGA TAG GAC CAG GGT ATC CC and GCT GCC TAT TAT TGG TAA GAG TGG; MOGi-cre (knock-in, 700 bp) TCC AAT TTA CTG ACC GTA CAC and CAT CAG CTA CAC CAG AGA CGG AAA TC; iDTR [30] (WT 600 bp, KI 845 bp) AAA GTC GCT CTG AGT TGT TAT, GGA GCG GGA GAA ATG GAT AAA GTC GCT CTG AGT TGT TAT, GGA GCG GGA GAA ATG GAT ATG, and AAT AGG AAC TTC GTC GAG AAT AGG AAC TTC GTC GAG C; $L u-$ ciferase (415 bp) TGT TGT TCC ATT CCA TCA CGG and ATC CAG ATC CAC AAC CTT CGC; and EYFP (200 bp) CTA TAT CAT GGC CGA CAA GC and ACT GGG TGC TCA GGT AGT GG.

\section{In vivo bioluminescence recordings and disease models}

oLucR mice were shaved and anesthetized, and light emission was recorded in vivo in an ultrasensitive IVIS 100 system (Caliper Life Science, Hopkinton, MA, USA) after intraperitoneal (i.p.) injection of $150 \mathrm{ng} / \mathrm{kg}$ of D-luciferin. The average photon-per-second recording over the full kinetics of the luciferase reaction was used as readout per mouse, per measurement.

For EAE experiments, 6- to 10-week-old mice were immunized subcutaneously with $200 \mu \mathrm{g}$ (each flank $100 \mu \mathrm{g}$ ) of myelin oligodendrocyte glycoprotein $\mathrm{MOG}_{35-55}$ peptide (MEVGWYRSPFSRVVHLYRNGK) emulsified in Complete Freund's Adjuvant (CFA; H37 Ra, Difco Laboratories, Franklin Lakes, NJ, USA), and injected i.p. the same day and at day 2 with $200 \mathrm{ng}$ pertussis toxin (Sigma-Aldrich, St. Louis, MO, USA). To deplete ODCs through DTx administration, 6- to 10-week-old oLucR/ diphtheria toxin receptor (DTR) animals were injected i.p. with 200 ng DTx (Merck, Darmstadt, Germany) daily over 7 days. EAE scoring and composite score of DTx-induced clinical disease was performed as in [11].

\section{Ex vivo CNS slices}

Animals were killed and perfused with phosphate-buffered saline (PBS), and CNS were collected and cut in half. From one half of the brain and spinal cord, approximately 1mm-thick slices were cut, bathed in $150 \mu \mathrm{g} / \mathrm{ml}$ luciferin, and immediately recorded for 2 min within an IVIS 100 system (Caliper Life Science).

\section{Luminometer assay}

Mouse tissue was dissolved in lysis buffer (Promega Corporation, Fitchburg, WI, USA). Protein content was quantified by the Bradford method (Bio-Rad, Munich, Germany) using BSA as protein standard. Lysates were 
adjusted to same protein content and analyzed according to manufacturer's guidelines in a black 96-well plate on a microplate luminometer (Victor2, Wallac, Freiburg, Germany).

\section{Immunostaining of cells after cytospin}

Cell sorting was carried out using a FACSAria III (BD Biosciences, San Jose, CA, USA). Dead cells were excluded using an Aqua Live/Dead staining reagent (Invitrogen, Carlsbad, CA, USA) and microglial/myeloid cells by positive staining for CD45 and CD11b. Sorted EYFP ${ }^{+}$ ODCs were spun onto glass slides $(600 \mathrm{rpm})$ (Shandon Southern Instruments, Sewickley, PA, USA). Cells were fixed in $4 \%$ paraformaldehyde (10 min), incubated with $4 \%$ normal goat serum in $0.2 \%$ Triton-PBS, and then immunostained. For primary antibodies, goat anti-GFP antibody (Rockland, Gilbertsville, PA, USA), mouse anti-CC1 (Calbiochem, Darmstadt, Germany), rabbit anti-PLP (Abcam, Cambridge, UK), and Isolectin b4-Alexa Fluor 568 (SigmaAldrich, St. Louis, MO, USA) were used as indicated and were detected with secondary anti-goat Alexa Fluor 488-, anti-mouse Alexa Fluor 405-, anti-rabbit Alexa Fluor 647conjugated antibody (all by Invitrogen, Carlsbad, CA, USA). Confocal microscopy was performed using a SP5 confocal laser microscopy (Leica, Wetzlar, Germany) and images analyzed using Imaris software (version 7.5.1; Bitplane, Zurich, Switzerland).

\section{Expression analysis}

Samples were homogenized in $1 \mathrm{ml}$ TRIZOL and incubated for $5 \mathrm{~min}$ at room temperature (RT). Two hundred microliters chloroform were added, incubated for $3 \mathrm{~min}$ at RT, and centrifuged at 8,000 rpm for $15 \mathrm{~min}$ at $4^{\circ} \mathrm{C}$. The RNA in the aqueous phase was precipitated by $500 \mu \mathrm{l}$ isopropyl alcohol (10-min incubation at RT and centrifugation at $800 \mathrm{rpm}$ for $10 \mathrm{~min}$ at $4^{\circ} \mathrm{C}$ ). The RNA pellet was washed with $1 \mathrm{ml} 75 \%$ ethanol. Samples were centrifuged at 5,000 rpm for $5 \mathrm{~min}$ at $4^{\circ} \mathrm{C}$, and the RNA pellet was dried for $15 \mathrm{~min}$ at RT and dissolved in $45 \mu \mathrm{l}$ RNase free water with $5 \mu \mathrm{l}$ of $10 \times$ incubation buffer and $1 \mu \mathrm{l}$ DNaseI. The samples were incubated at $37^{\circ} \mathrm{C}$ for $20 \mathrm{~min}$, and then, $1 \mu \mathrm{l} 0.5 \mathrm{M}$ EDTA was added, incubated at $75^{\circ} \mathrm{C}$ for $10 \mathrm{~min}$, and placed on ice. For FACSsorted cell samples, RNA was prepared using an RNeasy Plus Micro Kit (Qiagen, Valencia, CA, USA). One microliter random primers $(100 \mathrm{ng} / \mu \mathrm{l}), 1 \mu \mathrm{dNTP}(10 \mathrm{mM})$, $4 \mu \mathrm{l}$ first-strand buffer $(5 \times), 2 \mu \mathrm{l}$ DTT $(0.1 \mathrm{M})$, and $1 \mu \mathrm{l}$ RNase OUT were incubated for $2 \mathrm{~min}$ at $37^{\circ} \mathrm{C}$, and then,

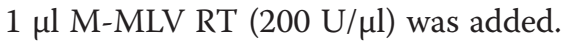

Five micrograms RNA were diluted in $10 \mu \mathrm{l}$ RNase free $\mathrm{H}_{2} \mathrm{O}$, heated to $65^{\circ} \mathrm{C}$ for $5 \mathrm{~min}$, and quenched on ice. Afterwards, $10 \mu \mathrm{l}$ Master Mix were added to the RNA, incubated $10 \mathrm{~min}$ at $21^{\circ} \mathrm{C}, 50 \mathrm{~min}$ at $37^{\circ} \mathrm{C}$, and finally $15 \mathrm{~min}$ at $70^{\circ} \mathrm{C}$. The cDNA was finally diluted $1: 10$.
Five microliters of cDNA per real-time reaction were mixed with $12.5 \mu \mathrm{l}$ SYBR Green, $6.5 \mu \mathrm{l} \mathrm{H}_{2} \mathrm{O}, 0.5 \mu \mathrm{l}$ FPrimer, and $0.5 \mu \mathrm{l}$ R-Primer for a total volume of $25 \mu \mathrm{l}$. The real-time (RT-PCR) was performed using a C1000 Touch Thermal Cycler (Bio-Rad CFX384 Real-Time System, Bio-Rad, Munich, Germany) with the following primers: $\beta$-actin, F: AGA GGG AAA TCG TGC GTG AC; R: CAA TAG TGA TGA CCT GGC CGT; Nestin, F: CAA GAA CCA CTG GGG TC, R: CCC TCC TGG TGA TTC CAC A; NG2, F: GTT GGG ATG CTT GCT GG, R: TGA AAG CTG CAG AAG CA; $M O G$, F: AAA TGG CAA GGA CCA AG, R: AGC AGG TGT AGC CTC CTT; OLIG1, F: ACC AAC GTT TGA GCT TGC TT, R: GGT TAA GGA CCA GCC TGT GA; $\beta$-Tubulin, F: TCG TGG AAT GGA TCC CCA AC; R: CTC CAT CTC GTC CAT GCC CT; and RNA polymerase II: CTG GTC CTT CGA ATC CGC ATC and GCT CGA TAC CCT GCA GGG TCA.

\section{Immunohistochemistry}

Mice were euthanized with $\mathrm{CO}_{2}$ and perfused with PBS. For cryostat sections, the tissue was fixed overnight with $4 \%$ PFA, cryoprotected in 30\% sucrose and frozen at $-80^{\circ} \mathrm{C}$. Frozen tissue was cut sagittally in $40-$ $\mu \mathrm{m}$-thick sections and stained with myelin basic protein (MBP)-specific (Dako, Glostrup, Denmark) and $\beta$-actinspecific (Biolegend, San Diego, CA) antibodies. Detection was accomplished using Alexa fluor-coupled secondary antibodies (Life Technologies, Karlsruhe, Germany). Sections were covered with Vectashield (Vector Laboratories, Burlingame, CA) and analyzed by confocal (Leica SP7, Leica, Wetzlar, Germany) microscope.

\section{Statistical analysis}

Unless otherwise indicated, data were displayed as mean \pm SEM, and statistical analysis performed by two-tailed Student's $t$ test using Excel software (Microsoft). Disease onset/bioluminescence increase correlation analysis was performed by Pearson's correlation coefficient analysis.

\section{Results}

\section{In vivo bioluminescence in the oLucR mouse model is} specific for mature ODC

The aim of our study was to investigate cellular dynamics of ODCs in an in vivo mouse model. As microfilament dynamics control the fine morphological changes during OPC migration, differentiation, and ODC myelination, the activation of the $\beta$-actin promoter was used as indirect readout of ODC plasticity. We generated a mouse model (oLucR) in which activity of the $\beta$-actin promoter can be monitored specifically within ODCs through luciferase expression. We achieved this by using a strain in which luciferase was driven by the $\beta$-actin promoter but its expression restricted to ODCs through 
excision of a loxP-flanked STOP cassette by the MOGicre strain specific for mature ODCs [28,29] (Figure 1a). Specificity and efficacy of the Cre-mediated recombination for terminally differentiated ODCs were tested by breeding the MOGi-cre mouse line with the EYFP reporter line, in which expression of the fluorescent protein EYFP was dependent on excision of a STOP cassette. Between $65 \%$ and $95 \%$ of $\mathrm{CC1}^{+}$, ODCs showed efficient locus recombination, depending on the CNS region (data not shown). To further characterize these cells, we FACS-sorted EYFP $^{+}$cells from dissociated CNS tissue of MOGi-cre x EYFP reporter mice. FACS-sorted ODCs appeared devoid of myelinated processes but remained alive (see 'Methods' section) and stained positive for PLP (myelin proteolipid protein) and CC1, both markers of mature ODCs (Figure 1b). Also, RT-PCR of sorted $\mathrm{EYFP}^{+}$cells revealed strong expression of the mature ODC marker gene MOG and no expression of the precursor markers NG2 or nestin (data not shown). We could thus confirm ex vivo that mature ODCs physically deprived of their myelin sheaths can indeed survive the insult $[23,25,26]$. Taken together, Cre activity in MOGicre mice was confirmed to be restricted to mature ODC.

In vivo bioluminescence in oLucR mice was visualized and quantified in an IVIS 100 system (Caliper Life Science) after i.p. injection of $150 \mathrm{ng} / \mathrm{kg}$ of luciferin. The luciferase signal was characterized by reproducible kinetics of photon emission showing an emission peak $10 \mathrm{~min}$ after luciferin injection (Figure 1c). Since minor photon emission was also detected from non-CNS areas (such as liver, tail, and paws), we recorded the luciferase signal from specific regions of interest (ROIs) covering the brain and spinal cord (Figure 1d). While faint bioluminescence signals from the liver and spleen were expected [28], photon emission from the tail and paws proved to be an in vivo artifact, as freshly dissected tails and limbs did not show any luciferase expression ex vivo (data not shown). As expected, absolute luciferase content in the CNS of oLucR mice increased during postnatal development reflecting ODC maturation and thereby MOG expression [31] (Figure 1e). Longitudinal analysis of the luciferase signal in adult oLucR mice showed stable luciferase-driven bioluminescence over extended time periods (up to 5 weeks), as a consequence of steady-state expression of $\beta$-actin gene in undisturbed myelin (Figure 1f). Taken together, we showed that the oLucR system was suitable to indirectly visualize the in vivo activity of the $\beta$ actin promoter within mature ODCs.

\section{DTx-mediated ODC death increases overall ODC $\beta$-actin promoter activity}

ODC death and demyelination are pathological hallmarks of several human CNS diseases such as certain hereditary and metabolic leukodystrophies and MS [1,32]. While it has been shown in several animal models that remyelination by differentiating OPCs follows myelin destruction [16], the behavior of the surviving mature ODC population in response to demyelinating stress or even death of nearby ODCs is still unclear. To investigate dynamics of the cytoskeleton in mature ODCs after demyelinating insults, we used the oDTR system [11] for experimental ODC death. We crossed oLucR mice to the iDTR strain [30] to obtain DTR expression specifically on $\mathrm{MOG}^{+} \mathrm{ODC}$. We recently showed that in the resulting oDTR system, DTx administration leads to a highly specific ablation of up to $60 \%$ of ODCs in disseminated white matter areas, followed by diffuse demyelination starting 1 week post administration (p.a.) [11]. In this model, surviving unaffected mature ODCs are often located scattered within demyelinated areas or in close proximity to damaged ODC neighbors [11].

We injected into these oLucR/oDTR and into control oLucR mice $200 \mathrm{ng}$ of DTx daily for a week. As a consequence of damage to the ODC population, DTx-treated oLucR/oDTR mice, but not oLucR control mice, presented with progressive and severe motor impairment starting 5 weeks p.a. (Figure 2a). In oLucR/oDTR mice, bioluminescence increased approximately threefold compared to the basal level during the first week of DTx administration (Figure $2 \mathrm{~b}$ ), parallel to the initial demyelination classically observed in the oDTR model [11]. To confirm that this signal peak was not the result of a damaged blood brain barrier (BBB) and thus easier access of luciferin to the brain parenchyma, we performed additional analyses. First, freshly prepared brain slices were placed in luciferin followed by ex vivo analysis of light emission (Figure 2c); second, total brain lysates were assessed by luminometer measurements at the end of the demyelination period (Figure 2d). Both assays confirmed increased luciferase expression in the CNS of DTx-treated oLucR/oDTR mice compared to controls. Furthermore, to support our observation obtained with the transgenic luciferase reporter system, we analyzed $\beta$-actin RNA levels in samples from different CNS areas. Since $\beta$-actin was the gene of interest in our analysis, we utilized transcript level of RNA polymerase II as housekeeping gene and internal control in this and following assays [33]. We found that, in contrast to the decrease in MOG expression (Figure 2e), $\beta$-actin was upregulated in all CNS regions during the initial period of increased in vivo luciferase activity (Figure 2f). Taken together, sterile ODC ablation led to increased ODCspecific, $\beta$-actin-driven bioluminescence, thus suggesting activation of cytoskeletal plasticity in ODCs upon demyelinating stressors.

\section{Increased activity of $\beta$-actin-driven luciferase at onset of autoimmune CNS inflammation}

DTx-mediated pathology derives from direct, sterile insults to ODCs [11]. Yet, the most common demyelinating 
a
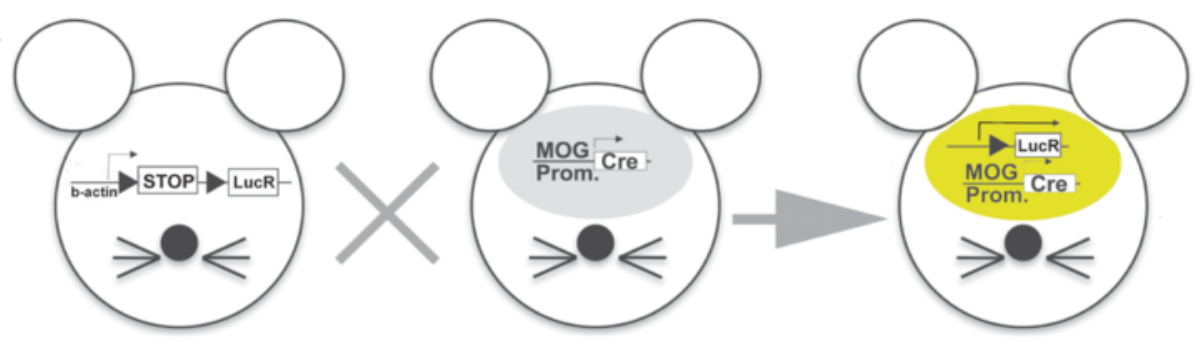

b

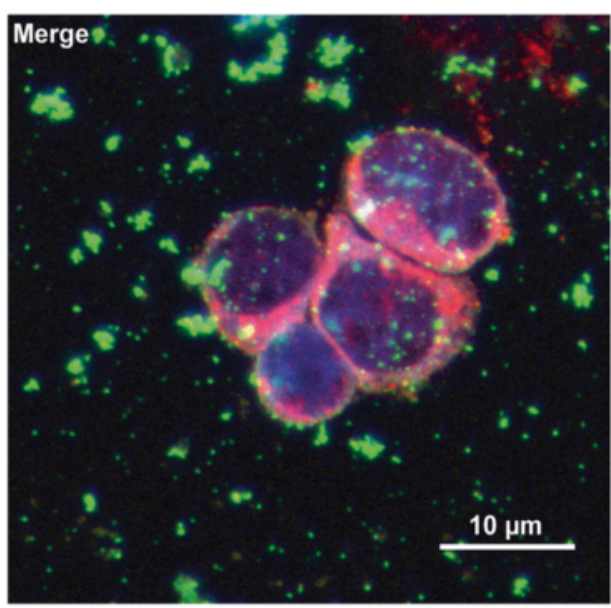

C

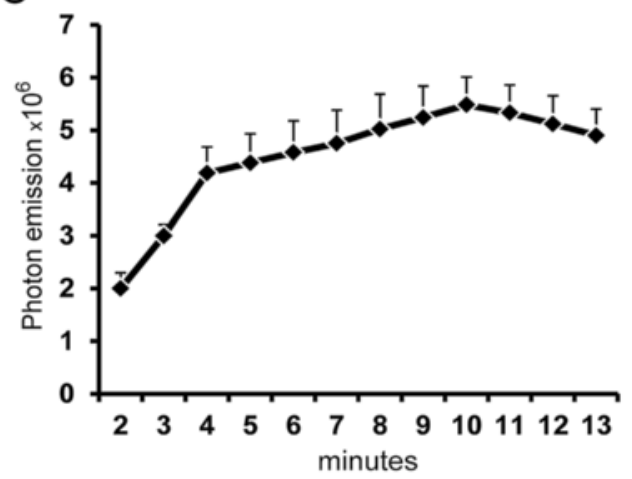

e

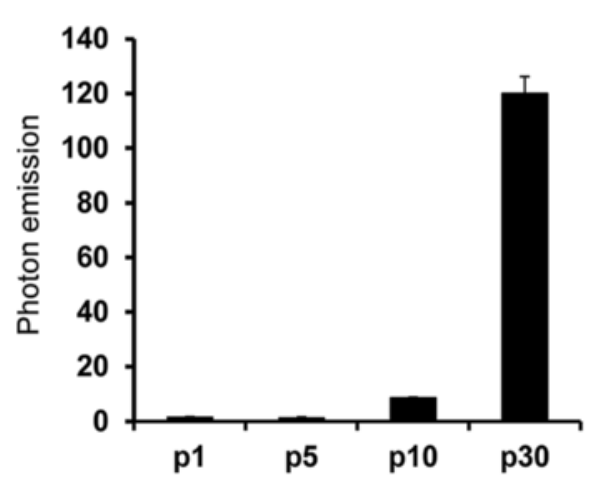

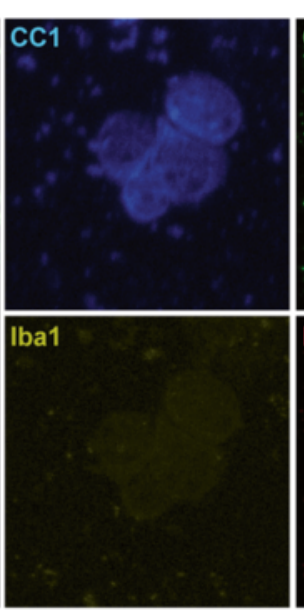
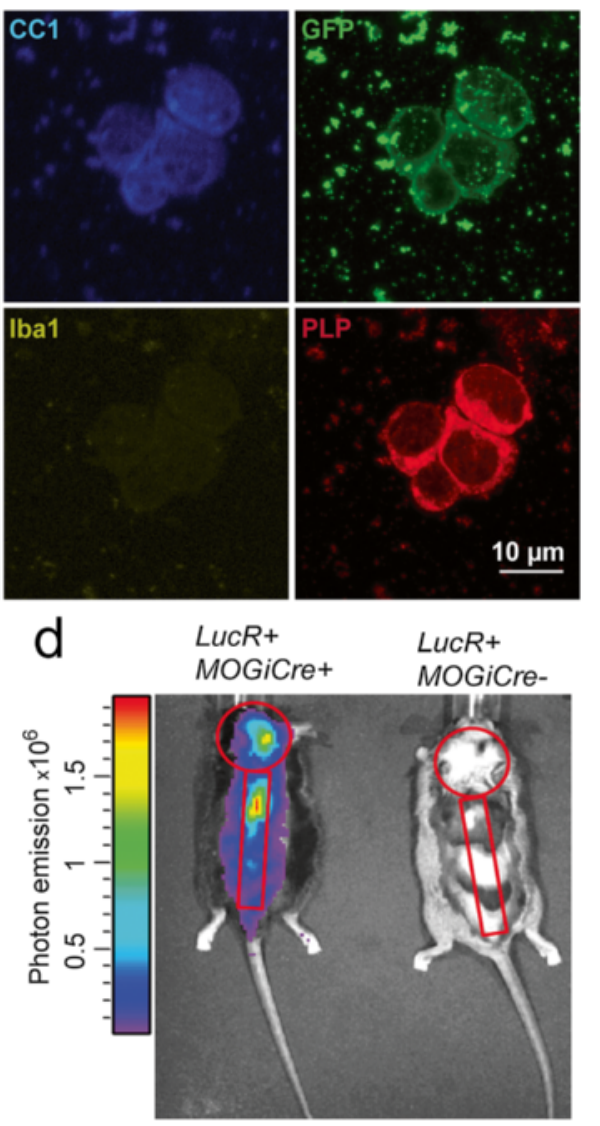

$\mathrm{f}$

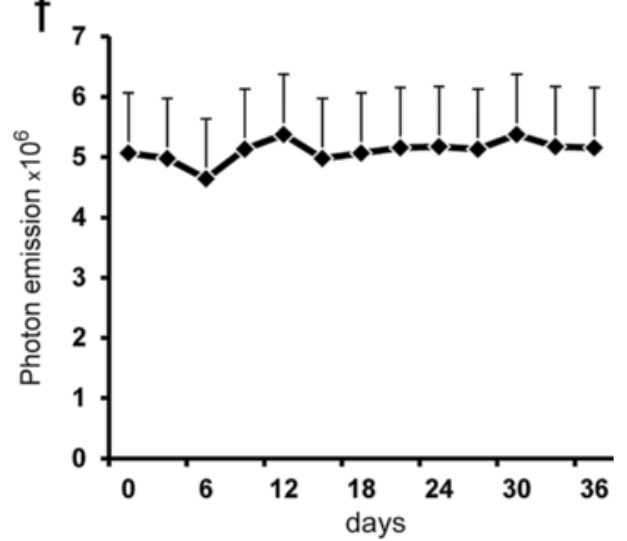

Figure 1 (See legend on next page.) 
(See figure on previous page.)

Figure 1 The oLucR mouse model shows CNS-specific in vivo bioluminescence. (a) ODC-specific expression of luciferase is achieved by crossing a Cre-inducible luciferase reporter mouse (left) to the MOGi-cre strain. (b) EYFP+ cells were sorted and stained with CC1-, GFP-, Iba1-, and PLP-specific antibodies following cytospin. Microglia/macrophage cells were excluded by positive CD45 and CD11b staining. Dead cells were excluded by Aqua Live/Dead staining reagent (Life Technologies). (c) Kinetics of photon emission acquired with an IVIS camera in anesthetized oLucR animals following intraperitoneal injection of $150 \mathrm{ng} / \mathrm{kg}$ of D-luciferin (mean $\pm \mathrm{SEM}, n=8$ ). (d) In vivo bioluminescence recorded in a representative oLuCR mouse (left) and a control LuCR animal where the STOP codon impedes luciferase expression (right). Shown in red are the specific regions of interest (ROIs) for signal acquisition. (e) The CNS from oLucR mice of the indicated ages were homogenized and analyzed in a luminometer assay. Photon emission of the lysates is shown (mean $\pm S E M, n=3$ ). (f) oLucR mice were injected with luciferin every 3 days and bioluminescence recorded from specific brain and spinal cord ROls over the course of 36 days (mean \pm SEM, $n=4$ ).

disease in humans is MS, in which an inflammatory process results in demyelination and axonal degeneration [34]. To assess whether immune-mediated damage to the CNS would induce an active plastic reaction of the mature ODC compartment, we measured the $\beta$-actin-driven bioluminescence response in oLucR mice in the EAE model. Mice were immunized against a myelin-derived peptide $\left(\mathrm{MOG}_{35-55}\right)$, leading to a $\mathrm{T}$ cell-mediated attack against CNS myelin and to direct and indirect insults to ODCs [9]. In this system, a variable degree of demyelination and cell death is observed, predominantly in the spinal cord and brain stem [1]. We thus recorded bioluminescence every 2 to 3 days in immunized and in naïve control
(oLucR) animals. After a transient increase in bioluminescence at day 2 post immunization (p.i.), we detected a sharp, approximately 12-fold increase in luciferase signal compared to baseline (Figure 3a,b). Such bioluminescence increase was observed consistently at the disease onset in MOG $_{35-55}$-immunized oLucR mice (Figure 3a,b) and was followed by a sharp decrease in bioluminescence almost reaching levels found before immunization and in naive animals (Figure 3b). Notably, the acute peak in bioluminescence consistently correlated with the beginning of clinical EAE signs independent of the exact time point of disease onset (Figure 3c). At the same time, bioluminescence in MOG-immunized oLucR mice that did not

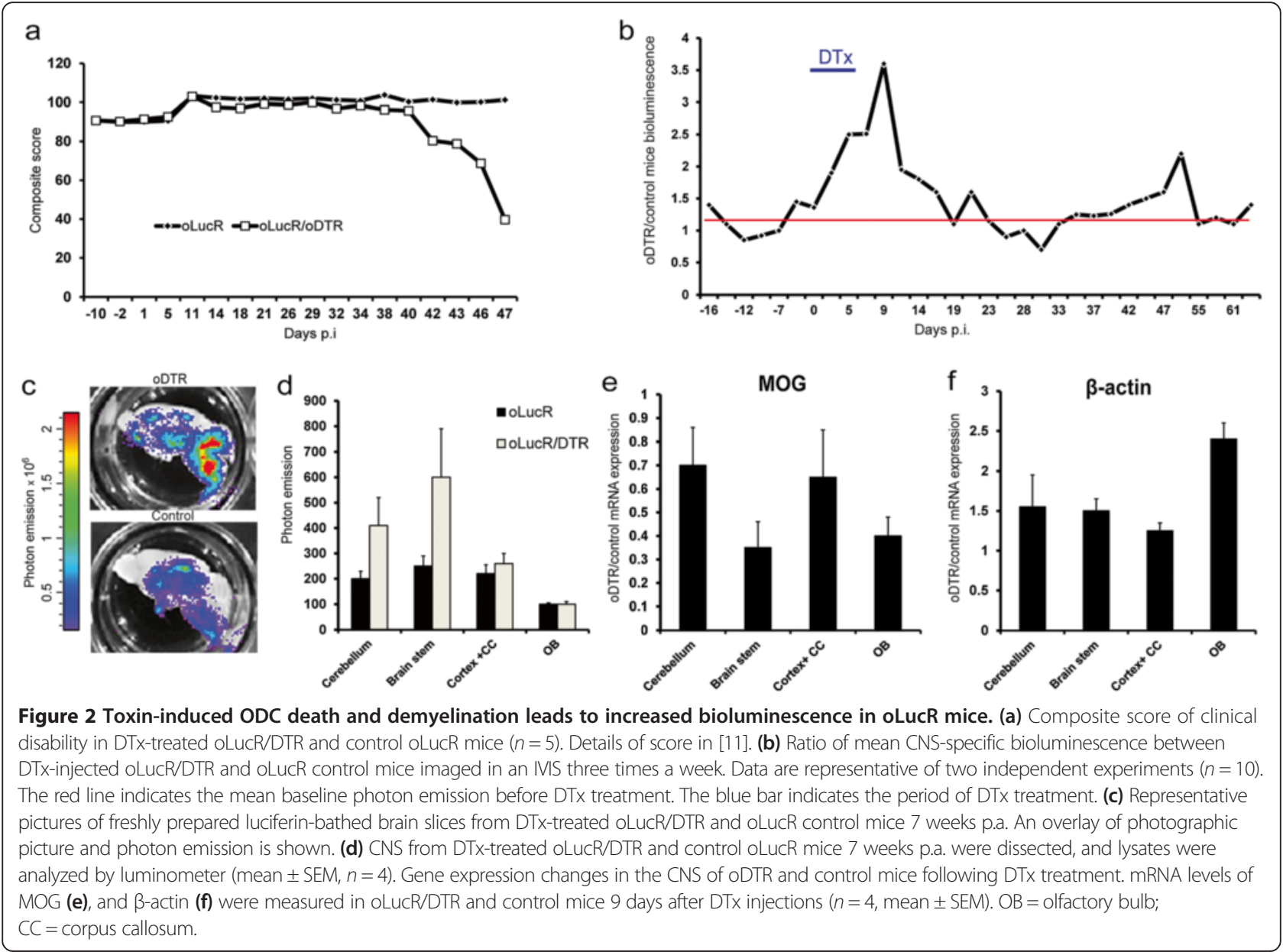



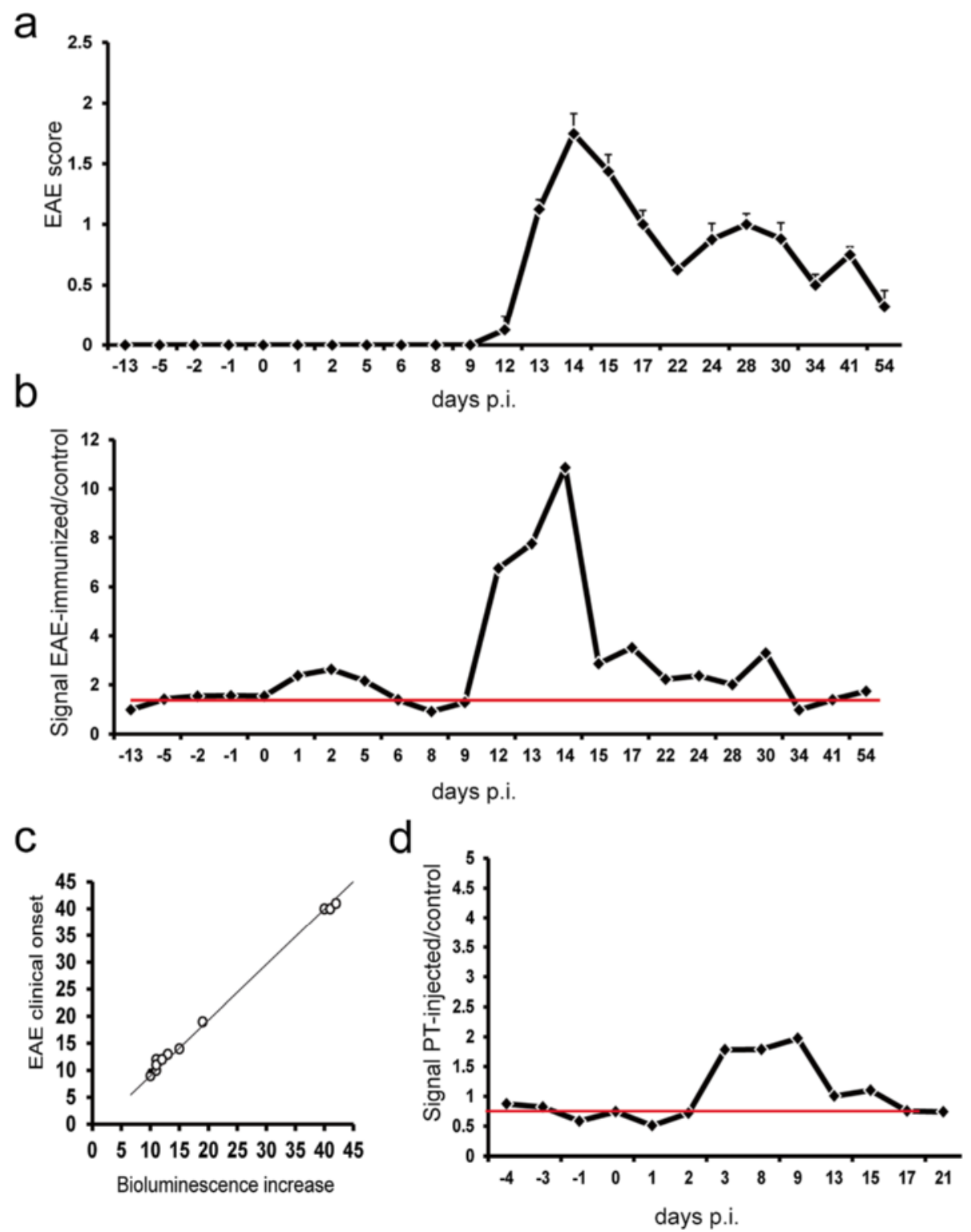

Figure 3 CNS autoimmune inflammation leads to increased bioluminescence in oLucR mice at clinical onset of disease. (a) EAE disease scores in MOG-immunized oLucR animals (mean \pm SEM, $n=5$ ). (b) Ratio of mean CNS-specific bioluminescence between MOG-immunized and control mice imaged in an IVIS every 2 to 3 days after immunization. Data are representative of three independent experiments $(n=15)$. The red line indicates the baseline photon emission before MOG immunization. (c) Linear correlation between day of clinical onset and increase of bioluminescence in MOG-immunized oLucR mice $(n=15$, Pearson's correlation coefficient $=0.9989)$. The three mice with disease onset around day 40 did not develop clinically overt EAE after the initial EAE induction and therefore were re-immunized with MOG peptide after 1 month. (d) Ratio of mean CNS-specific bioluminescence between PT-treated and control mice. oLucR mice were injected with $300 \mathrm{ng}$ PT at day 0 and 2 and bioluminescence acquired over time $(n=5)$. The red line indicates baseline photon emission before PT administration.

develop overt clinical paralysis remained unchanged over time (data not shown). This also indicated that not MOG immunization per se, but injury to ODCs and subsequent neurological deficits were closely related to actin/cytoskeleton ODC dynamics. We then tested whether increase in bioluminescence could have been the result of higher access of luciferin to the CNS. First, we investigated whether the adjuvant pertussis toxin (PT) alone, known to transiently increase BBB permeability [35,36], could affect in vivo bioluminescence in our model. PT administration in oLucR animals resulted in a low and transient increase in luciferase signal (Figure 3d), similar to the minor bioluminescence increase observed in the EAE experiment 1 to 5 days post immunization (Figure $3 \mathrm{~b}$ ). Second, we immunized oLucR animals with $\mathrm{MOG}_{35-55}$ in CFA and analyzed luciferin-bathed brain slices every other day from 
day 5 to day 15 p.i. Ex vivo bioluminescence followed similar dynamics as the one observed in vivo, with increased luciferase signals detected in all CNS regions of mice developing clinical EAE, albeit predominantly in the severely affected spinal cord (Figure 4a,b). In contrast, CNS slices from immunized mice that were found not to develop the disease did not show any increase in ex vivo bioluminescence (data not shown). Third, in vitro analysis of luciferase activity of CNS lysates revealed increased reporter levels especially in the spinal cord and brain stem of EAE-induced animals compared to controls (Figure 4c). Thus, intrinsic damage-related dynamical responses of the cytoskeleton within the ODC population account for the major bioluminescence changes observed in oLucR mice. To further investigate the nature of the bioluminescence increase at EAE onset, we immunized oLucR animals and analyzed gene expression in the spinal cord at day 11 p.i. We observed a decrease in ODC markers such as MOG and Olig1 (Figure 5a,b), while $\beta$-actin levels were strongly increased specifically in oLucR animals induced with EAE (Figure 5c). Notably, 5 days after clinical EAE onset, at the beginning of the ameliorating remitting phase, $\beta$-actin expression decreased almost reaching control levels (Figure $5 \mathrm{~d}$ ). As several invading immune cells and resident activated glial cells might contribute to the observed dynamics in whole-tissue $\beta$ actin transcription, we FACS-isolated mature EYFP ${ }^{+}$ ODCs from naïve and MOG-immunized EYFP reporter mice and analyzed expression level of cytoskeletal genes within these cells. In this assay, $\beta$-actin levels appeared unchanged in ODCs during the course of EAE (Figure 5e). However, $\beta$-actin mRNA is known to be transported to distal sites and leading edges within cells $[37,38]$. Our flow cytometry purification approach necessarily shed distal myelin processes from isolated ODCs (Figure 1b). Accordingly, immunostaining of spinal cord sections showed increased presence of $\beta$-actin in myelin structures and ODC processes at disease onset compared to controls (Figure 5e). To investigate other changes in cytoskeleton gene expression, we then analyzed the major component of ODC cytoskeleton, $\beta$-tubulin, for which distal mRNA transport has not been described. Our analysis indeed revealed significant upregulation of $\beta$-tubulin expression in mature ODCs at clinical onset of EAE compared to control naïve mice (Figure $5 \mathrm{f}$ ). Altogether, in vivo and ex vivo analysis of oLucR mice indicates that CNS inflammation induces drastic changes in cytoskeletal genes within the ODC population.

\section{Discussion}

ODCs were originally thought to provide mere structural requirements for saltatory signal transduction of neurons. Only in recent years, they found increasing recognition as fundamental supporters of neuronal metabolism and survival and have thus obtained attention for their active role in many neurodegenerative pathologies [2,3,10]. Mature ODC death and demyelination directly promote the development and accumulation of clinical disabilities within demyelinating diseases such as MS, leukodystrophies, and respective animal models $[11,32]$. Following such pathological processes, stem cells and OPCs enable a functional healing process through remyelination of insulted CNS areas. The current paradigm holds that during such insults and subsequent remyelination, postmitotic ODCs remain passive victims of damage [16]. In contrast to this traditional viewpoint, different studies support the hypothesis that mature ODCs actively respond to parenchymal stress [39] and maintain some degree of structural

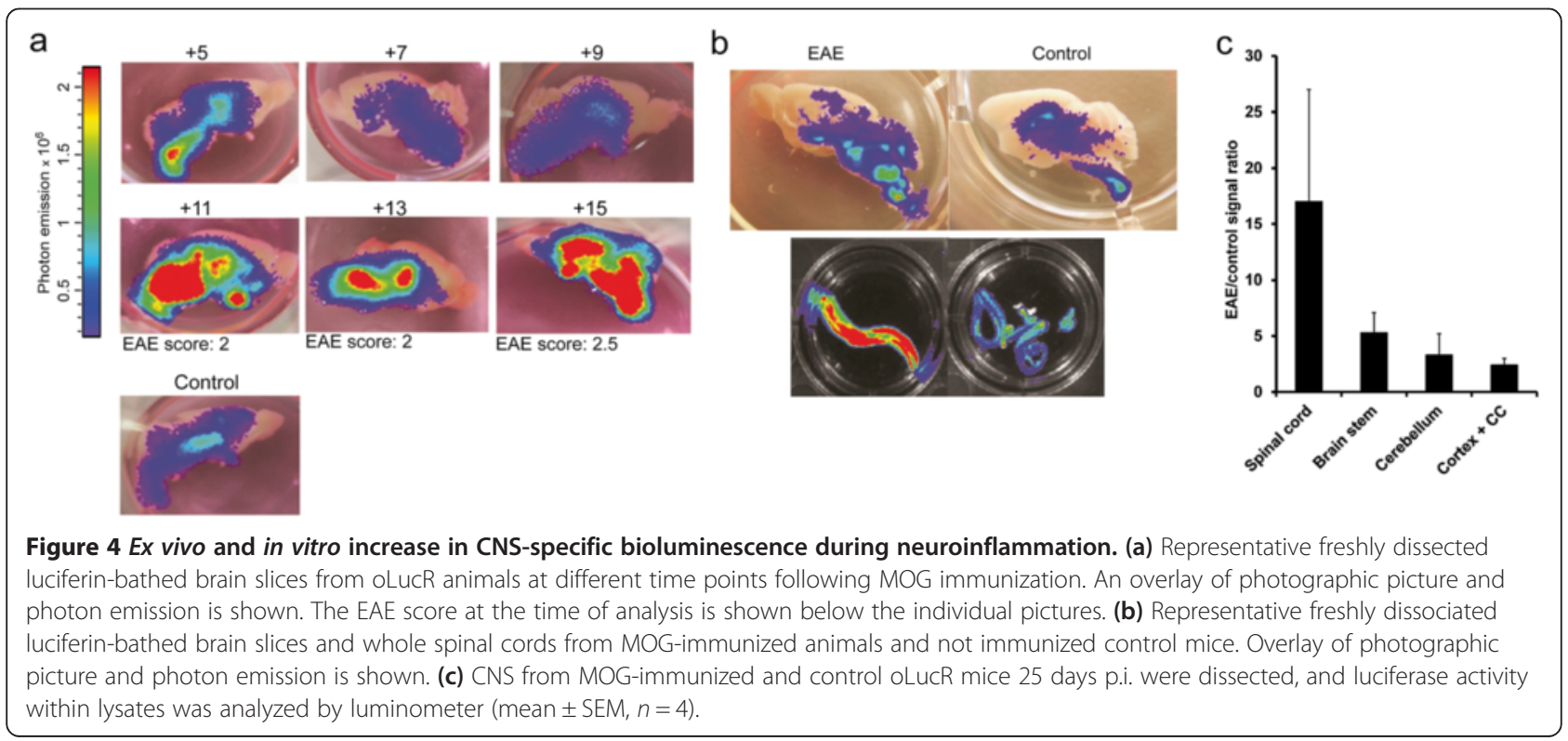




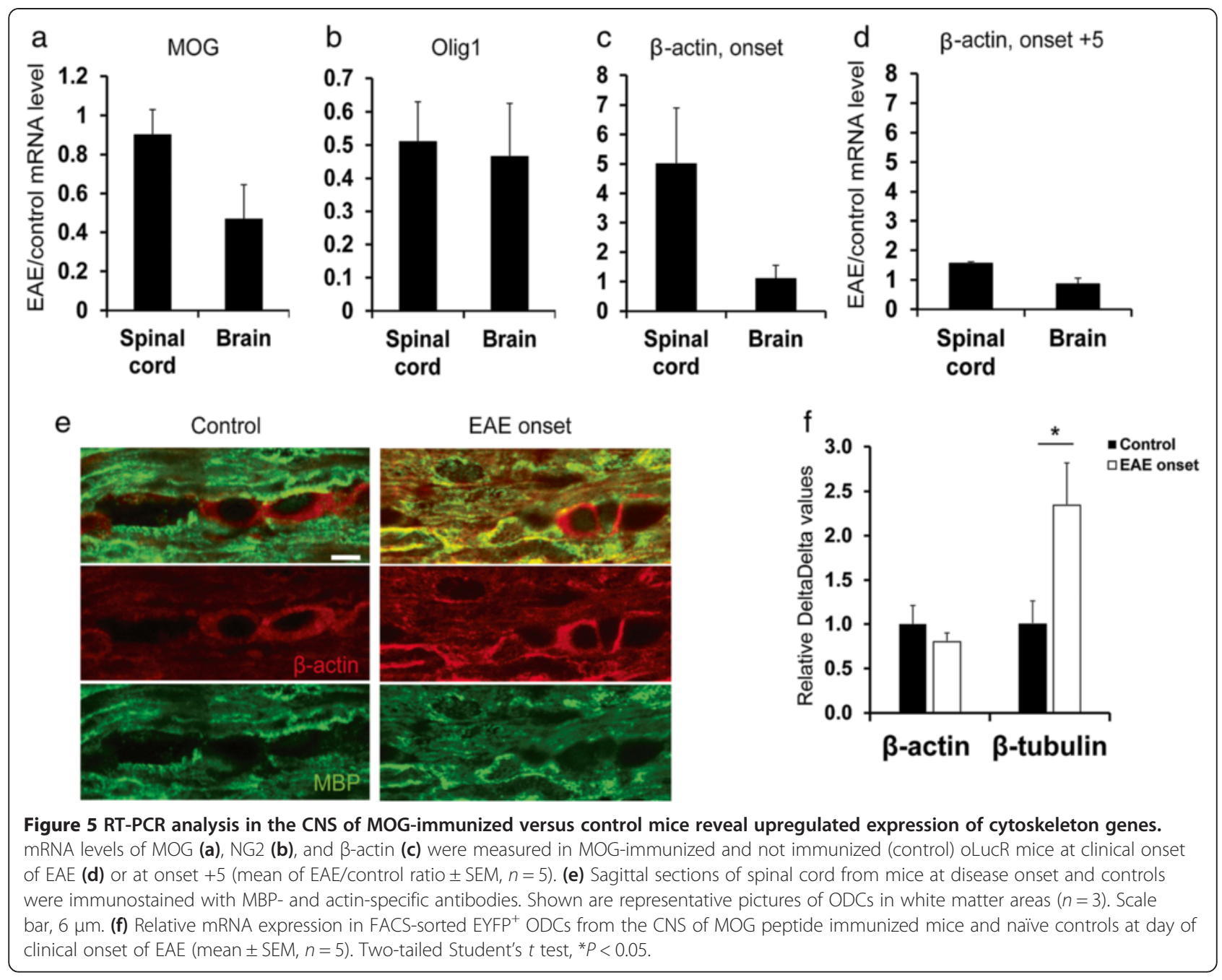

plasticity potentially enabling not only ODC survival during neuroinflammation but also complex dynamic adaptation upon environmental cues [21-25]. Unfortunately, little is known about the in vivo response and potential functional plasticity of mature ODCs upon demyelinating insults. Underlying the complex cytoarchitecture of ODCs, $\beta$-actin and $\beta$-tubulin are necessary candidates as main players in ODC and myelin dynamics. Even though both have been traditionally considered housekeeping genes, their transcriptional control can be substantially affected during cell proliferation, activation, differentiation, membrane fragmentation, and in the context of different pathological conditions [33,40-42]. We therefore chose the $\beta$-actin promoter in combination with luciferase as a readout to investigate the fine in vivo dynamics of mature ODCs. Given its low background, high quantum yield, and good tissue penetration, the luciferase reporter is widely used in macroscopic in vivo measurements [43]. In our oLucR mouse model, luciferase expression is controlled by the $\beta$-actin promoter and restricted to MOG- expressing ODCs by a Cre/loxP system. Luciferase activity in these mice increased during CNS development followed by a stable, reproducible signal intensity in adult animals, as expected from the known sequence of myelination events [31]. Cre/loxP recombination was highly effective and specific for mature, $\mathrm{PLP}^{+} \mathrm{MOG}^{+} \mathrm{NG}^{\text {negative }}$ ODCs. Thus, oLucR mice allowed us to follow in vivo bioluminescence changes specifically from the terminally differentiated ODC population.

In this study, different experimental insults to myelin and ODCs led to a consistent augmentation of ODCspecific bioluminescence in oLucR mice. First, in the oDTR model [11], widespread ODC death through DTx administration in oLucR/oDTR mice transiently albeit consistently increased in vivo bioluminescence. Second, a strong rise in luciferase signal was observed after autoimmune inflammatory myelin and ODC damage, correlating with the clinical disease onset of EAE in oLucR mice. Such variations in luciferase levels appeared intrinsic to the demyelinating CNS as ex vivo and in vitro 
analysis of freshly cut CNS slices and CNS protein extracts confirmed these in vivo results. Altogether, despite the possibility of signal loss due to ODC death and reduction in ODC numbers, demyelinating conditions led to a consistent increase in the ODC-specific luciferase reporter signal. The post-lesion timing of such bioluminescence changes clearly rules out any possible signal contribution from recruited/differentiated OPCs. PT adjuvant treatment alone, known to increase BBB permeability, resulted in a transient and low signal augmentation, suggesting that enhanced luciferin access to the CNS can account only for a small proportion of the observed fluctuations. We did not formally exclude a direct 'activatory' effect of PT on ODCs [44], but this seems unlikely due to the delayed occurrence of enhanced in vivo bioluminescence in the EAE model. Nonetheless, the relatively fast decline of in vivo bioluminescence following the rapid increases in luciferase emission at clinical onset of EAE or during DTx administration in oLucR/DTR mice remains somewhat puzzling. In DTxinjected oLucR/oDTR mice, the drastic drop in bioluminescence can be easily ascribed to the massive ODC degeneration observed 1 week post DTx administration [11]. However, death of luciferase ${ }^{+}$ODCs alone cannot explain the bioluminescence decrease following the emission peak observed during EAE, as ODC death in such paradigm is neither massive nor sharply-timed. Rather, such emission kinetics might indicate that mature ODC 'activation' represents a transient process which closely mirrors the strong, destructive inflammation observed in the early phases of EAE. Also, increased tissue hypoxia in the inflamed spinal cord $[45,46]$ could directly contribute to the decrease in bioluminescence following disease onset, as luciferase activity is highly dependent on the amount of oxygen available [47]. Overall, the consistent increase of in vivo and in vitro bioluminescence upon immune- or toxin-mediated demyelination/ODC damage suggests that luciferase ${ }^{+}$mature ODCs exhibit some kind of stress-induced acute activation of the cytoskeleton encoding genes within and surrounding experimental demyelinating lesions.

Accordingly, total $\beta$-actin expression was higher in the CNS of EAE-induced oLucR and DTx-treated oLucR/ oDTR mice than in control mice. We have previously shown that in the oDTR model, no lymphocyte infiltration, OPC recruitment, microglia or astroglia activation can be observed 1 week after induction of demyelination [11]. Therefore, in the DTx-treated oLucR/oDTR mouse model, the early increase in actin transcription can be ascribed to a transient mobilization of mature luciferase ${ }^{+}$ ODCs, which were not killed by DTx. Surviving mature ODCs in the oDTR model are generally located in close proximity to damaged cells and within demyelinating CNS areas [11]. ODCs could thus sense pathological changes of dying neighboring ODCs through soluble mediators or via gap junctions within the glial syncytium [48]. However, in oLucR mice at clinical onset of EAE, the observed increase in CNS $\beta$-actin expression is likely affected by inflammatory processes such as leukocyte infiltration and glial activation. We thus performed immunostaining of myelin and $\beta$-actin in white matter areas at onset of EAE and observed stronger expression of $\beta$-actin in ODC processes and attached myelin sheaths. Additionally, we repeated RNA expression analysis on purified mature ODCs through FACS-sorting of EYFP ${ }^{+}$ ODCs but found no difference in $\beta$-actin expression upon onset of clinical EAE in sorted ODCs. This apparent conundrum could result from our technical approach of cell purification, as our RT-PCR data can only provide information on mRNA content of the ODC cell body, but not on distally transported mRNAs, which are highly enriched in myelin (that is, $M B P$ mRNA $[49,50]$ ). Such mechanism of distal transport and translation has been indeed extensively described for $\beta$-actin mRNA $[37,38]$ and allows higher polymerization rate of microfilaments and increased cell motility in the leading edge of dynamic cellular structures. It is thus likely that mature ODCs enhance $\beta$-actin expression and mRNA distal transport in vivo upon demyelinating stress. Different from $\beta$-actin mRNA particles, $\beta$-tubulin mRNA is not transported to distal cellular compartments, as microtubules are very rare in thin villi of mature cells and leading edges of myelinating progenitors compared to microfilaments [51]. Through our expression analysis on purified $\mathrm{MOG}^{+}$cells, we could thus show for the first time that $\beta$-tubulin is significantly increased in mature ODC cell bodies at clinical onset of CNS inflammation. Altogether, demyelination/ODC damage leads to upregulation of the main component of the cytoskeleton, $\beta$-tubulin, and hints toward an enhanced distal distribution of upregulated $\beta$-actin mRNA in activated mature ODCs.

\section{Conclusions}

Our study addresses a long-standing issue on the biology of mature ODCs within demyelinating CNS: the current perception of postmitotic ODCs as simple passive victims of insults to the myelin. This notion is put under discussion by studies describing different degrees of structural plasticity of mature ODCs in different in vivo and in vitro experimental scenarios and the presence of significant mechanisms of myelin remodeling existing throughout adulthood [5,18-26,52]. Accordingly, the oLucR model allowed us to 1 ) indirectly show through the activity of a $\beta$-actin-driven reporter gene and 2) directly prove through immunohistochemistry and expression analysis of damaged CNS/sorted cells, that mature ODCs experience a dynamic in vivo activation of the cytoskeleton possibly leading to reorganization of myelin sub-structures. Our 
observations thus suggest that mature ODCs can actively respond to various demyelinating $\mathrm{CNS}$ insults, increase their cytoskeletal plasticity in vivo upon different types of parenchymal stress, and may thus constitute potential targets for therapies aimed at supporting neuroprotection/ remyelination.

\section{Abbreviations}

CNS: central nervous system; DTx: diphtheria toxin; EAE: experimental autoimmune encephalomyelitis; i.p.: intraperitoneal; MS: multiple sclerosis; ODC: oligodendrocyte; OPC: oligodendrocyte precursor; PT: pertussis toxin.

\section{Competing interests}

The authors declare that they have no competing interests.

\section{Authors' contributions}

$\mathrm{TB}$ and $\mathrm{BB}$ conceived the experimental system. $\mathrm{GL}, \mathrm{AB}, \mathrm{BS}$, and PP carried out the experiments and analyzed the data. TB, AW, and BB supervised the work. $\mathrm{GL}$ and TB co-wrote the manuscript. All authors read and approved the final manuscript.

\section{Authors' information}

BB and TB jointly directed the study.

\section{Acknowledgements}

We are grateful for the support by the technician and mouse teams in Zurich and Munich. We thank Andreas Diefenbach for providing ROSA-EYFP mice and Anton Berns for providing the LucR mice. We thank Vinko Tosevski, Florian Mair, the Flow Cytometry, and the Microscopy Facility of the University of Zurich for support. We thank Martin Kerschensteiner for helping with the manuscript preparation. This work was supported by the Swiss State Secretariat for Education and Research SFB within the framework of the European Union COST action BM603 and grants by the Swiss Multiple Sclerosis Society and the Hertie Foundation to TB. The funders had no role in the study design, data collection and analysis, decision to publish, or preparation of the manuscript.

\section{Author details}

${ }^{1}$ Institute of Experimental Immunology, University of Zurich, Winterthurerstrasse 190, Zürich 8057, Switzerland. ${ }^{2}$ Institute of Clinical Neuroimmunology, LMU Universität München, Marchioninistrasse 17, Munich 81377, Germany. Institute for Molecular Medicine, University Medical Center of the Johannes Gutenberg, University of Mainz, Obere Zahlbacher Str. 67, Mainz 55131, Germany. ${ }^{4}$ Institute for Medical Microbiology, Immunology and Hygiene, Technische Universität München, Trogerstrasse 30, 80675 Munich, Germany. Institute of Laboratory Animal Science, VetSuisse, University of Zurich, Winterthurerstrasse 190, Zurich 8057, Switzerland.

\section{Received: 7 October 2014 Accepted: 20 February 2015}

\section{Published online: 02 April 2015}

\section{References}

1. Bradl M, Lassmann H. Oligodendrocytes: biology and pathology. Acta Neuropathol. 2010;119(1):37-53.

2. Funfschilling UL, Supplie M, Mahad D, Boretius S, Saab AS, Edgar J, et al. Glycolytic oligodendrocytes maintain myelin and long-term axonal integrity. Nature. 2012;485(7399):517-21.

3. Lee Y, Morrison BM, Li Y, Lengacher S, Farah MH, Hoffman PN, et al. Oligodendroglia metabolically support axons and contribute to neurodegeneration. Nature. 2012:487(7408):443-8.

4. Watkins TA, Emery B, Mulinyawe S, Barres BA. Distinct stages of myelination regulated by gamma-secretase and astrocytes in a rapidly myelinating CNS coculture system. Neuron. 2008;60(4):555-69.

5. Snaidero N, Möbius W, Czopka T, Hekking LH, Mathisen C, Verkleij D, et al. Myelin membrane wrapping of CNS axons by PI(3,4,5)P3-dependent polarized growth at the inner tongue. Cell. 2014;16(156(1-2)):277-90.

6. Pfeiffer SE, Warrington AE, Bansal R. The oligodendrocyte and its many cellular processes. Trends Cell Biol. 1993;3(6):191-7.

7. Haber M, Vautrin S, Fry EJ, Murai KK. Subtype-specific oligodendrocyte dynamics in organotypic culture. Glia. 2009;57(9):1000-13.
8. Boggs JM, Rangaraj G, Heng YM, Liu Y, Harauz G. Myelin basic protein binds microtubules to a membrane surface and to actin filaments in vitro: effect of phosphorylation and deimination. Biochim Biophys Acta. 2011;1808(3):761-73.

9. McTigue DM, Tripathi RB. The life, death, and replacement of oligodendrocytes in the adult CNS. J Neurochem. 2008;107(1):1-19.

10. Nave KA. Myelination and support of axonal integrity by glia. Nature. 2010:468(7321):244-52

11. Locatelli G, Wörtge S, Buch T, Ingold B, Frommer F, Sobottka B, et al. Primary oligodendrocyte death does not elicit anti-CNS immunity. Nat Neurosci. 2012;15(4):543-50.

12. Gensert JM, Goldman JE. Endogenous progenitors remyelinate demyelinated axons in the adult CNS. Neuron. 1997;19(1):197-203.

13. Keirstead HS, Blakemore WF. Identification of post-mitotic oligodendrocytes incapable of remyelination within the demyelinated adult spinal cord. J Neuropathol Exp Neurol. 1997;56(11):1191-201.

14. Nunes MC, Roy NS, Keyoung HM, Goodman RR, McKhann 2nd G, Jiang L, et al. Identification and isolation of multipotential neural progenitor cells from the subcortical white matter of the adult human brain. Nat Med. 2003;9(4):439-47.

15. Fancy SP, Zhao C, Franklin RJ. Increased expression of Nkx2.2 and Olig2 identifies reactive oligodendrocyte progenitor cells responding to demyelination in the adult CNS. Mol Cell Neurosci. 2004;27(3):247-54.

16. Franklin RJ, French-Constant C. Remyelination in the CNS: from biology to therapy. Nat Rev Neurosci. 2008;9(11):839-55.

17. Sim FJ, Zhao C, Franklin RJ. The age-related decrease in CNS remyelination efficiency is attributable to an impairment of both oligodendrocyte progenitor recruitment and differentiation. J Neurosci. 2002;22(7):2451-9.

18. Arenella $L S$, Herndon RM. Mature oligodendrocytes. Division following experimental demyelination in adult animals. Arch Neurol. 1984;41(11):1162-5.

19. Ludwin SK. Proliferation of mature oligodendrocytes after trauma to the central nervous system. Nature. 1984;308(5956):274-5.

20. Ludwin SK, Bakker DA. Can oligodendrocytes attached to myelin proliferate? J Neurosci. 1988:8(4):1239-44.

21. Scolding NJ, Morgan BP, Houston WA, Linington C, Campbell AK, Compston DA. Vesicular removal by oligodendrocytes of membrane attack complexes formed by activated complement. Nature. 1989;339(6226):620-2.

22. Fernandez-Gamba A, Leal MC, Maarouf CL, Richter-Landsberg C, Wu T, Morelli L, et al. Collapsin response mediator protein-2 phosphorylation promotes the reversible retraction of oligodendrocyte processes in response to non-lethal oxidative stress. J Neurochem. 2012;121(6):985-95.

23. Makinodan M, Okuda-Yamamoto A, Ikawa D, Toritsuka M, Takeda T, Kimoto $\mathrm{S}$, et al. Oligodendrocyte plasticity with an intact cell body in vitro. PLoS ONE. 2013;8(6):e66124.

24. Knapp PE. Injury stimulates outgrowth and motility of oligodendrocytes grown in vitro. Exp Cell Res. 1997;234(1):7-17.

25. Wolswijk G. Oligodendrocyte survival, loss and birth in lesions of chronicstage multiple sclerosis. Brain. 2000;123(Pt 1):105-15.

26. Chang A, Tourtellotte WW, Rudick R, Trapp BD. Premyelinating oligodendrocytes in chronic lesions of multiple sclerosis. N Engl J Med. 2002;346(3):165-73.

27. Richter-Landsberg C. Organization and functional roles of the cytoskeleton in oligodendrocytes. Microsc Res Tech. 2001:52(6):628-36.

28. Lyons SK, Meuwissen R, Krimpenfort P, Berns A. The generation of a conditional reporter that enables bioluminescence imaging of Cre/loxPdependent tumorigenesis in mice. Cancer Res. 2003;63(21):7042-6.

29. Hövelmeyer N, Hao Z, Kranidioti K, Kassiotis G, Buch T, Frommer F, et al. Apoptosis of oligodendrocytes via Fas and TNF-R1 is a key event in the induction of experimental autoimmune encephalomyelitis. J Immunol. 2005; 175:5875-84.

30. Buch T, Heppner FL, Tertilt C, Heinen TJ, Kremer M, Wunderlich FT, et al. A Cre-inducible diphtheria toxin receptor mediates cell lineage ablation after toxin administration. Nat Methods. 2005;2(6):419-26.

31. Solly SK, Thomas JL, Monge M, Demerens C, Lubetzki C, Gardinier MV, et al. Myelin/oligodendrocyte glycoprotein (MOG) expression is associated with myelin deposition. Glia. 1996;18(1):39-48.

32. Benarroch EE. Oligodendrocytes: susceptibility to injury and involvement in neurologic disease. Neurology. 2009;72(20):1779-85.

33. Rubie C, Kempf K, Hans J, Tilton B, Georg T, Brittner B, et al. Housekeeping gene variability in normal and cancerous colorectal, pancreatic, esophageal, gastric and hepatic tissues. Mol Cell Probes. 2005;19(2):101-9. 
34. Compston A, Coles A. Multiple sclerosis. Lancet. 2008;372(9648):1502-17.

35. Hofstetter HH, Shive HL, Forsthuber TG. Pertussis toxin modulates the immune response to neuroantigens injected in incomplete Freund's adjuvant: induction of Th1 cells and experimental autoimmune encephalomyelitis in the presence of high frequencies of Th2 cells. J Immunol. 2002;169(1):117-25.

36. Bruckener KE, el Baya A, Galla HJ, Schmidt MA. Permeabilization in a cerebral endothelial barrier model by pertussis toxin involves the PKC effector pathway and is abolished by elevated levels of CAMP. J Cell Sci. 2003;116(Pt 9):1837-46.

37. Condeelis J, Singer HS. How and why does $\beta$-actin mRNA target? Biol Cell. 2005;97:97-110.

38. Ma B, Savas JN, Yu M-S, Culver BP, Chao MV, Tanese N. Huntingtin mediates dendritic transport of $\beta$-actin mRNA in rat neurons. Sci Rep. 2001;1:140. doi:10.1038.

39. Zeis T. Schaeren-Wiemers. Lame ducks or fierce creatures? The role of oligodendrocytes in multiple sclerosis. J Mol Neurosci. 2008;35(1):91-100.

40. Ruan W, Lai M. Actin, a reliable marker of internal control? Clin Chim Acta. 2007;385(1-2):1-5.

41. Matthews PR, Eastwood SL, Harrison PJ. Reduced myelin basic protein and actin-related gene expression in visual cortex in schizophrenia. PLOS ONE. 2012;7(6):e38211.

42. Jung J, Cai W, Lee HK, Pellegatta M, Shin YK, Jang SY, et al. Actin polymerization is essential for myelin sheath fragmentation during Wallerian degeneration. J Neurosci. 2011;31(6):2009-15

43. Weissleder $R$, Ntziachristos $V$. Shedding light onto live molecular targets. Nat Med. 2003;9(1):123-8

44. Moorman SJ. The inhibition of motility that results from contact between two oligodendrocytes in vitro can be blocked by pertussis toxin. Glia. 1996;16(3):257-65.

45. Nathoo NS, Agrawal Y, Wu S, Haylock-Jacobs WW Yong, Foniok T, Barnes S, et al. Susceptibility-weighted imaging in the experimental autoimmune encephalomyelitis model of multiple sclerosis indicates elevated deoxyhemoglobin, iron deposition and demyelination. Mult Scler. 2012;19(6):721-31.

46. Davies AL, Desai RA, Bloomfield PS, Mclntosh PR, Chapple KJ, Linington C, et al. Neurological deficits caused by tissue hypoxia in neuroinflammatory disease. Ann Neurol. 2013;74:815-25.

47. Contag $\mathrm{CH}$, Bachmann $\mathrm{MH}$. Advances in in vivo bioluminescence imaging of gene expression. Annu Rev Biomed Eng. 2002;4:235-60.

48. Magnotti LM, Goodenough DA, Paul DL. Functional heterotypic interactions between astrocyte and oligodendrocyte connexins. Glia. 2011;59(1):26-34.

49. Ainger K, Avossa D, Morgan F, Hill SJ, Barry C, Barbarese E, et al. Transport and localization of exogenous myelin basic protein mRNA microinjected into oligodendrocytes. J Cell Biol. 1993;123(2):431-41.

50. Müller C, Bauer NM, Schäfer I, White R. Making myelin basic protein - from mRNA transport to localized translation. Front Cell Neuroscience. 2013;7:Article 169.

51. Richter-Landsberg C. The oligodendroglia cytoskeleton in health and disease. J Neurosc Res. 2000;59:11-8.

52. Young KM, Psachoulia K, Tripathi RB, Dunn SJ, Cossell L, Attwell D, et al. Oligodendrocyte dynamics in the healthy adult CNS: evidence for myelin remodeling. Neuron. 2013;77(5):873-85.

\section{Submit your next manuscript to BioMed Central and take full advantage of:}

- Convenient online submission

- Thorough peer review

- No space constraints or color figure charges

- Immediate publication on acceptance

- Inclusion in PubMed, CAS, Scopus and Google Scholar

- Research which is freely available for redistribution 